\title{
Nonrelativistic string theory sigma model and its canonical formulation
}

\author{
Josef Klusoñ ${ }^{\mathrm{b}}$ \\ Department of Theoretical Physics and Astrophysics, Faculty of Science, Masaryk University, Kotlářská 2, 61137 Brno, Czech Republic
}

Received: 15 November 2018 / Accepted: 21 January 2019 / Published online: 4 February 2019

(C) The Author(s) 2019

\begin{abstract}
This paper is devoted to the canonical analysis of non-linear sigma model that describes motion of nonrelativistic string on stringy Newton-Cartan background. We determine structure of constraints of this string and compare resulting Hamiltonian with previous proposal of nonrelativistic string on stringy Newton-Cartan background.
\end{abstract}

\section{Introduction and summary}

In $[1,2]$ non-relativistic string theory with Galilean invariant global symmetry was proposed. ${ }^{1}$ This theory is described by two-dimensional quantum field theory which is well defined and which contain fields that describe dynamics of string in target space-time together with additional fields which are crucial for consistency of string theory. It is important to stress that the target space-time, where the string propagates corresponds to flat space-time invariant under global stringy Galilean symmetry. The characteristic property of non-relativistic string theory is that there is no Riemannian metric in the target space. In fact, non-relativistic string theory provides a quantization of stringy non-relativistic spacetime geometry in the same way as relativistic string theory provides quantization of Riemannian geometry. Natural question is whether we can formulate non-linear sigma model that describes string propagation on a non-relativistic target space-time structure. As was shown recently in [17] the appropriate geometry corresponds to so-called stringy Newton-Cartan geometry [18]. ${ }^{2}$

The action proposed in [17] is very interesting and certainly deserves further study. In particular, it would be very nice to find Hamiltonian form of this action and analyze its

\footnotetext{
${ }^{1}$ For related works, see for example [3-16].

${ }^{2}$ For related works, that analyse point particles or extended objects in Newton-Cartan geometry or its stringy generalizations, see for example [19-25].

be-mail: klu@physics.muni.cz
}

relation to Hamiltonian that was proposed recently in [26]. ${ }^{3}$ The goal of this paper is to perform such an analysis. It turns out that the canonical analysis of the action proposed in [17] is rather non-trivial due to the complicated structure of the target space-time and also thanks to the presence of additional world-sheet fields that are needed for the consistency of theory. Since these fields are non-dynamical we find that their conjugate momenta are the primary constraints of the theory. Then requirement of the preservation of these primary constraints implies secondary constraints that together with primary constraints are second class constraints. Hence they can be explicitly solved with very interesting result. In more details, in order to find Hamiltonian formulation of the action proposed in [17] we should find metric inverse to the boost invariant metric that defines string sigma model in stringy Newton-Cartan gravity. It turns out that crucial object for construction of such a metric is matrix valued Newton potential which is natural generalization of Newton potential defined in Newton-Cartan geometry. Then we will be able to find corresponding Hamiltonian and diffeomorphism constraints and we show that they are the first class constraints. As a next step we proceed to the solution of the second class constraints. It turns out that when we solve these constraints and insert this result into the original Hamiltonian constraint we find that the resulting constraint agrees with the Hamiltonian constraint found in [26] which is very nice consistency check of both approaches. Note that the Hamiltonian found in [26] was derived with the help of the limiting procedure that defines Newton-Cartan geometry from the relativistic one [27]. In more details, we start with the Hamiltonian for relativistic string in general background. Then we used the form of the metric [27] that leads to the stringy Newton-Cartan background in the limit when parameter $\omega$ goes in infinity. We argued that in order to cancel divergence in the Hamiltonan constraint of non-relativistic string when this procedure is

\footnotetext{
3 The Hamiltonian analysis of non-relativistic string in the background with flat longitudinal dimension was performed previously in [14].
} 
performed, we should include background NSNS two form in the special form that was chosen in such a way to obtain finite Hamiltonian constraint. In principle this procedure is not unique and hence the agreement between the Hamiltonian constraint found in [26] and derived here shows that the ansatz introduced in [26] is the correct one.

Finally we determine Lagrangian from corresponding Hamiltonian and we find that it agrees exactly with the Lagrangian found in [18] which is again very nice consistency check. Let us outline our results and suggest possible extension of this work. We find canonical structure of non-linear sigma model proposed recently in [17]. We determine all constraints and we identify Hamiltonian and spatial diffeomorphism constraints and calculate Poisson brackets between them. We also determine second class constraints and perform their explicit solutions which gives the Hamiltonian constraint that agrees with the constraint found in [26].

The next important step in our canonical formulation of non-relativistic string theory is to perform analysis of T-duality since, as was shown in [17], T-duality of nonrelativistic string theory is more complex than in case of its relativistic version. This analysis is currently in progress.

The structure of this paper is following. In the next Sect. (2) we review basic facts about stringy Newton-Cartan geometry and non-linear sigma model defined on it, following [17]. Then in Sect. (3) we perform canonical analysis of this theory. Finally in Sect. (4) we explicitly solve second class constraint and determine corresponding Hamiltonian.

\section{Non-linear sigma model on stringy Newton-Cartan geometry}

In this section we define stringy Newton-Cartan geometry, following [17]. Let $\mathcal{M}$ is $D+1$ dimensional manifold and let $\mathcal{T}_{p}$ is tangent space at point $p$. We decompose $\mathcal{T}_{p}$ into longitudinal directions indexed by $A=0,1$ and transverse directions with $A^{\prime}=2, \ldots, d-1$. Two dimensional foliation of $\mathcal{M}$ is defined by generalized clock function $\tau_{\mu}{ }^{A}$ that is also known as longitudinal vielbein field that satisfies a constraint

$D_{\mu} \tau_{\nu}{ }^{A}-D_{\nu} \tau_{\mu}{ }^{A}=0$,

where $D_{\mu}$ is covariant derivative with respect to the longitudinal Lorentz transformations acting on index $A$. Let us also introduce transverse vielbein field $E_{\mu}{ }^{A^{\prime}}$. We further introduce projective inverse $\tau_{A}^{\mu}$ and $E_{A^{\prime}}^{\mu}$ that are defined as

$E_{\mu}^{A^{\prime}} E_{B^{\prime}}^{\mu}=\delta_{B^{\prime}}^{A^{\prime}}, \quad \tau_{A}^{\mu} \tau_{\mu}^{B}=\delta_{A}^{B}$,

$\tau_{\mu}^{A} \tau_{A}^{\nu}+E_{\mu}^{A^{\prime}} E_{A^{\prime}}^{\nu}=\delta_{\mu}^{v}$,

$\tau_{A}^{\mu} E_{\mu}^{A^{\prime}}=0, \quad \tau_{\mu}^{A} E_{A^{\prime}}^{\mu}=0$.
Let $\Sigma_{A}^{A^{\prime}}$ is a parameter of string Galilei boost transformations. Then various components of NC geometry transform in the following way

$\delta_{\Sigma} \tau_{\mu}{ }^{A}=0, \quad \delta_{\Sigma} E_{\mu}^{A^{\prime}}=-\tau_{\mu}^{A}{ }^{A}{ }_{A}^{A^{\prime}}$,

$\delta_{\Sigma} \tau_{A}^{\mu}=E_{A^{\prime}}^{\mu} \Sigma_{A}^{A^{\prime}}, \quad \delta_{\Sigma} E_{A^{\prime}}^{\mu}=0$.

From vielbein we can construct longitudinal metric $\tau_{\mu \nu}=$ $\tau_{\mu}{ }^{A} \tau_{\nu}{ }^{B} \eta_{A B}$ and transverse metric $h^{\mu \nu}=E_{A^{\prime}}^{\mu} E^{v}{ }_{B^{\prime}} \delta^{A^{\prime} B^{\prime}}$ that are invariant under string Galilean boost transformations.

It is clear that in order to define string moving in stringy Newton-Cartan background we need transverse tensor $H_{\mu \nu}$ that is invariant under the string Galilei boost. It turns out that this can be done when we introduce gauge field $m_{\mu}^{A}$ and we can construct boost invariant tensor

$H_{\mu \nu}=E_{\mu}^{A^{\prime}} E_{\nu}{ }^{{ }^{\prime}} \delta_{A^{\prime} B^{\prime}}+\left(\tau_{\mu}{ }^{A} m_{\nu}{ }^{B}+\tau_{\nu}{ }^{A} m_{\mu}{ }^{B}\right) \eta_{A B}$.

In conclusion, $\tau_{\mu}{ }^{A}, E_{\mu}^{A^{\prime}}$ and $m_{\mu}^{A}$ defines stringy NewtonCartan geometry.

Now we are ready to proceed to the string sigma model that was introduced in [17]. An important point is that this model is relativistic on two-dimensional world-sheet and hence it should be defined on the Riemann surface $\Sigma$. It turns out that this action contains world-sheet scalars $x^{\mu}$ that parameterize an embedding string into target space time together with two worlds-sheet scalars that we denote as $\lambda$ and $\bar{\lambda}$. These fields are needed for the realization of string Galilei symmetry on the world-sheet theory.

Now we will be more explicit. Let $\sigma^{\alpha}, \alpha=0,1$ parameterize world-sheet surface $\Sigma$. The sigma model is endowed with two dimensional world-sheet metric $\gamma_{\alpha \beta}$ and we introduce two dimensional vielbein $e_{\alpha}^{a}, a=0,1$ so that

$\gamma_{\alpha \beta}=e_{\alpha}^{a} e_{\beta}^{b} \eta_{a b}$

where $\eta_{a b}=\operatorname{diag}(-1,1)$. Using light-cone coordinates for the flat index $a$ on the world-sheet tangent space we define

$e_{\alpha} \equiv e_{\alpha}^{0}+e_{\alpha}{ }^{1}, \quad \bar{e}_{\alpha} \equiv e_{\alpha}^{0}-e_{\alpha}^{1}$.

We can also use light-cone coordinates for the flat index $A$ on the space-time tangent space $\mathcal{T}_{p}$ and define

$\tau_{\mu} \equiv \tau_{\mu}^{0}+\tau_{\mu}^{1}, \quad \bar{\tau}_{\mu}=\tau_{\mu}^{0}-\tau_{\mu}^{1}$

Then we are ready to write sigma model for non-relativistic string on an arbitrary string Newton-Cartan geometry, nonrelativistic Kalb-Ramond B-field $B_{\mu \nu}$ and dilaton field $\phi$ in the form [17]

$$
\begin{aligned}
S= & -\frac{T}{2} \int d^{2} \sigma\left(\sqrt{-\gamma} \gamma^{\alpha \beta} \partial_{\alpha} x^{\mu} \partial_{\beta} x^{\nu} H_{\mu \nu}\right. \\
& \left.+\epsilon^{\alpha \beta}\left(\lambda e_{\alpha} \tau_{\mu}+\bar{\lambda} \bar{e}_{\alpha} \bar{\tau}_{\mu}\right) \partial_{\beta} x^{\mu}\right)
\end{aligned}
$$




$$
\begin{aligned}
& -\frac{T}{2} \int d^{2} \sigma \epsilon^{\alpha \beta} \partial_{\alpha} x^{\mu} \partial_{\beta} x^{\nu} B_{\mu \nu} \\
& +\frac{1}{4 \pi} \int d^{2} \sigma \sqrt{-h} R \phi
\end{aligned}
$$

where $\gamma=\operatorname{det} \gamma_{\alpha \beta}, \gamma^{\alpha \beta}$ is inverse to $\gamma_{\beta \alpha}, R$ is scalar curvature of $\gamma_{\alpha \beta}$ and $T$ is string tension. Further, $\partial_{\alpha} x^{\mu}=\frac{\partial}{\partial \sigma^{\alpha}} x^{\mu}$. In what follows we restrict to the case of constant dilaton field so that the last term on the second line in (8) is total derivative and will be ignored. It is important to stress that $\lambda$ and $\bar{\lambda}$ are world-sheet scalars under change of wold-sheet coordinates $\sigma^{\prime \alpha}(\sigma)$. Explicitly, under such transformations we have

$\gamma_{\alpha \beta}^{\prime}\left(\sigma^{\prime}\right)=\frac{\partial \sigma^{\gamma}}{\partial \sigma^{\prime \alpha}} \frac{\partial \sigma^{\delta}}{\partial \sigma^{\prime \beta}} \gamma_{\gamma \delta}(\sigma), \quad \lambda^{\prime}\left(\sigma^{\prime}\right)=\lambda(\sigma)$,

$\bar{\lambda}^{\prime}\left(\sigma^{\prime}\right)=\bar{\lambda}(\sigma), \quad x^{\prime \mu}\left(\sigma^{\prime}\right)=x^{\mu}(\sigma)$.

Further, $\epsilon^{\alpha \beta}$ is Levi-Chivita symbol defined as $\epsilon^{01}=$ $-\epsilon^{10}=1$. Finally, we restrict ourselves to the case of the constant dilaton field so that the last term in (8) is topological invariant and will be ignored in what follows.

After this review of string sigma model in stringy NewtonCartan background we now proceed to its Hamiltonian formulation.

\section{Hamiltonian formulation of string in stringy Newton-Cartan background}

The presence of two dimensional vielbeins $e_{\alpha}^{a}$ makes the analysis slightly complicated and hence it is important to choose suitable parametrization. To do this we use convention introduced in $[28,29]$. Explicitly, let us define $e_{\alpha}$ and $\bar{e}_{\alpha}$ as

$e_{\alpha}^{0}=\frac{1}{2}\left(e_{\alpha}+\bar{e}_{\alpha}\right), \quad e_{\alpha}^{1}=\frac{1}{2}\left(e_{\alpha}-\bar{e}_{\alpha}\right)$.

Then it is easy to see that $\gamma_{\alpha \beta}=e_{\alpha}^{a} e_{\beta}^{b} \eta_{a b}$ has the form

$\gamma_{\alpha \beta}=-\frac{1}{2}\left(e_{\alpha} \bar{e}_{\beta}+\bar{e}_{\alpha} e_{\beta}\right)$

and also $\gamma=\operatorname{det} \gamma_{\alpha \beta}$ is equal to

$\gamma=-\frac{1}{4}\left(e_{0} \bar{e}_{1}-\bar{e}_{0} e_{1}\right)^{2}$.

Then inverse metric $\gamma^{\alpha \beta}$ has components

$$
\begin{aligned}
\gamma^{00} & =\frac{4 e_{1} \bar{e}_{1}}{\left(e_{0} \bar{e}_{1}-\bar{e}_{0} e_{1}\right)^{2}}, \quad \gamma^{11}=\frac{4 e_{0} \bar{e}_{0}}{\left(e_{0} \bar{e}_{1}-\bar{e}_{0} e_{1}\right)^{2}}, \\
\gamma^{01} & =-2 \frac{e_{0} \bar{e}_{1}+e_{1} \bar{e}_{0}}{\left(e_{0} \bar{e}_{1}-\bar{e}_{0} e_{1}\right)^{2}} .
\end{aligned}
$$

As the next step we introduce following variables $[28,29]$

$\xi=\ln \left(-e_{1} \bar{e}_{1}\right), \quad \epsilon=\frac{1}{2} \ln \left(-\frac{e_{1}}{\bar{e}_{1}}\right), \quad \Gamma^{+}=\frac{e_{0}}{e_{1}}$,

$\Gamma^{-}=-\frac{\bar{e}_{0}}{\bar{e}_{1}}$

with following inverse relation

$e_{1}=e^{\frac{1}{2}(\xi+2 \epsilon)}, \quad \bar{e}_{1}=e^{\frac{1}{2}(\xi-2 \epsilon)}$,

$e_{0}=\Gamma^{+} e^{\frac{1}{2}(\xi+2 \epsilon)}, \quad \bar{e}_{0}=-\Gamma^{-} e^{\frac{1}{2}(\xi-2 \epsilon)}$

and hence we obtain

$$
\begin{aligned}
\sqrt{-\gamma} \gamma^{00} & =-\frac{2}{\Gamma^{+}+\Gamma^{-}}, \quad \sqrt{-\gamma} \gamma^{11}=\frac{2 \Gamma^{+} \Gamma^{-}}{\Gamma^{+}+\Gamma^{-}}, \\
\sqrt{-\gamma} \gamma^{01} & =\frac{\Gamma^{+}-\Gamma^{-}}{\Gamma^{+}+\Gamma^{-}} .
\end{aligned}
$$

With the help of these relations we rewrite the action (8) into the form

$$
\begin{aligned}
S= & T \int d^{2} \sigma\left(\frac{1}{\Gamma^{+}+\Gamma^{-}} \dot{x}^{\mu}-\Gamma^{+} x^{\prime \mu}\right)\left(\dot{x}^{\nu}+\Gamma^{-} x^{\prime \nu}\right) H_{\mu \nu} \\
& -\frac{T}{2} \int d^{2} \sigma\left(\lambda \Gamma^{+} e^{\frac{1}{2}(\xi+2 \epsilon)} \tau_{\mu} x^{\prime \mu}-\lambda e^{\frac{1}{2}(\xi+2 \epsilon)} \tau_{\mu} \dot{x}^{\mu}\right) \\
& -\frac{T}{2} \int d^{2} \sigma\left(-\bar{\lambda} \Gamma^{-} e^{\frac{1}{2}(\xi-2 \epsilon)} \bar{\tau}_{\mu} x^{\prime \mu}-\bar{\lambda} e^{\frac{1}{2}(\xi-2 \epsilon)} \bar{\tau}_{\mu} \dot{x}^{\mu}\right) \\
& -T \int d^{2} \sigma \dot{x}^{\mu} x^{\prime \nu} B_{\mu \nu},
\end{aligned}
$$

where $\dot{x}^{\mu} \equiv \frac{\partial x^{\mu}}{\partial \sigma^{0}}, x^{\prime \mu}=\frac{\partial x^{\mu}}{\partial \sigma^{1}}$. From the form of the action (17) we see that it is natural to perform rescaling of $\lambda, \bar{\lambda}$ as

$\lambda^{+}=\lambda e^{\frac{1}{2}(\xi+2 \epsilon)}, \quad \lambda^{-}=\bar{\lambda} e^{\frac{1}{2}(\xi-2 \epsilon)}$

and hence the action (17) has the form

$$
\begin{aligned}
S= & T \int d^{2} \sigma \frac{1}{\Gamma^{+}+\Gamma^{-}}\left(\dot{x}^{\mu}-\Gamma^{+} x^{\prime \mu}\right)\left(\dot{x}^{\nu}+\Gamma^{-} x^{\prime \nu}\right) H_{\mu \nu} \\
& -T \int d^{2} \sigma \dot{x}^{\mu} x^{\prime \nu} B_{\mu \nu} \\
& -\frac{T}{2} \int d^{2} \sigma\left(\lambda^{+} \Gamma^{+} \tau_{\mu} x^{\prime \mu}-\lambda^{+} \tau_{\mu} \dot{x}^{\mu}\right) \\
& +\frac{T}{2} \int d^{2} \sigma\left(\lambda^{-} \Gamma^{-} \bar{\tau}_{\mu} x^{\prime \mu}+\lambda^{-} \bar{\tau}_{\mu} \dot{x}^{\mu}\right) .
\end{aligned}
$$

Before we proceed to the canonical formalism we would like to analyze the action (19) in more details. Let us determine equation of motion for $\lambda^{+}$and $\lambda^{-}$that follow from (19)

$\Gamma^{+} \tau_{\mu} x^{\prime \mu}-\tau_{\mu} \dot{x}^{\mu}=0, \quad \Gamma^{-} \bar{\tau}_{\mu} x^{\prime \mu}+\bar{\tau}_{\mu} \dot{x}^{\mu}=0$.

If we combine these equations together we obtain

$\Gamma^{+} \Gamma^{-}=-\frac{\tau_{\tau \tau}}{\tau_{\sigma \sigma}}, \quad \Gamma^{+}-\Gamma^{-}=2 \frac{\tau_{\tau \sigma}}{\tau_{\sigma \sigma}}$, 
where

$\tau_{\alpha \beta}=\tau_{\mu \nu} \partial_{\alpha} x^{\mu} \partial_{\beta} x^{\nu}, \quad \tau_{\mu \nu}=\tau_{\mu}^{A} \tau_{\nu}{ }^{B} \eta_{A B}$.

Equation (21) can be solved as

$\Gamma^{-}=\frac{-\tau_{\tau \sigma}+\sqrt{-\operatorname{det} \tau}}{\tau_{\sigma \sigma}}, \quad \Gamma^{+}=\frac{\tau_{\tau \sigma}+\sqrt{-\operatorname{det} \tau}}{\tau_{\sigma \sigma}}$.

Inserting this result into the action (19) we finally obtain

$$
\begin{aligned}
S= & -\frac{T}{2} \int d^{2} \sigma \sqrt{-\operatorname{det} \tau} \tau^{\alpha \beta} H_{\mu \nu} \partial_{\alpha} x^{\mu} \partial_{\beta} x^{\nu} \\
& -T \int d^{2} \sigma \dot{x}^{\mu} x^{\prime \mu} B_{\mu \nu}
\end{aligned}
$$

which corresponds to the non-relativistic string action as was formulated in [18].

Let us now proceed to the canonical formalism. From (19) we obtain following momenta $p_{\mu}$ conjugate to $x^{\mu}$

$$
\begin{aligned}
p_{\mu}= & T \frac{1}{\Gamma^{+}+\Gamma^{-}}\left(2 \dot{x}^{\nu}+\left(\Gamma^{-}-\Gamma^{+}\right) x^{\prime \nu}\right) H_{\nu \mu}-T B_{\mu \nu} x^{\prime \nu} \\
& +\frac{T}{2} \lambda^{+} \tau_{\mu}+\frac{T}{2} \lambda^{-} \bar{\tau}_{\mu},
\end{aligned}
$$

or equivalently

$$
\begin{aligned}
p_{\mu} & -T \frac{\Gamma^{-}-\Gamma^{+}}{\Gamma^{+}+\Gamma^{-}} H_{\mu \nu} x^{\prime \nu}+T B_{\mu \nu} x^{\prime \nu}-\frac{T}{2} \lambda^{+} \tau_{\mu}-\frac{T}{2} \lambda^{-} \bar{\tau}_{\mu} \\
& =\frac{2 T}{\Gamma^{+}+\Gamma^{-}} H_{\mu \nu} \dot{x}^{\nu} .
\end{aligned}
$$

Remaining conjugate momenta are primary constraints of the theory

$$
\begin{aligned}
& p_{+}^{\Gamma}=\frac{\partial L}{\partial \dot{\Gamma}^{+}} \approx 0, \quad p_{-}^{\Gamma}=\frac{\partial L}{\partial \dot{\Gamma}^{-}} \approx 0, \\
& p_{+}^{\lambda}=\frac{\partial L}{\partial \dot{\lambda}^{+}} \approx 0, \quad p_{-}^{\lambda}=\frac{\partial L}{\partial \dot{\lambda}^{-}} \approx 0 .
\end{aligned}
$$

Now using (25) we obtain Hamiltonian density in the form

$$
\begin{aligned}
\mathcal{H}= & p_{\mu} \dot{x}^{\mu}-\mathcal{L}=\frac{T}{\Gamma^{+}+\Gamma^{-}}\left(\dot{x}^{\mu} H_{\mu \nu} \dot{x}^{\nu}+\Gamma^{+} \Gamma^{-} H_{\mu \nu} x^{\prime \mu} x^{\prime \nu}\right) \\
& +\frac{T}{2} \lambda^{+} \Gamma^{+} \tau_{\mu} x^{\prime \mu}-\frac{T}{2} \lambda^{-} \Gamma^{-} \tau_{\mu} x^{\prime \mu}
\end{aligned}
$$

Of course, this is not correct form of the Hamiltonian density since it does not depend on canonical variables $p_{\mu}, x^{\mu}$. In order to express it in the right form we have to find relation between $\dot{x}^{\mu}$ and $p_{\mu}$. In order to solve this problem let us observe that we have following relation

$H_{\mu \rho} h^{\rho \sigma} H_{\sigma v}=H_{\mu \nu}+\tau_{\mu}^{A} \Phi_{A B} \tau_{v}{ }^{B}$,

where we defined matrix valued Newton potential $\Phi_{A B}$ as

$$
\begin{aligned}
& \Phi_{A B}=-\tau_{A}^{\sigma} m_{\sigma}^{C} \eta_{C B}-\eta_{A C} m_{\rho}^{C} \tau_{B}^{\rho} \\
& +\eta_{A C} m_{\rho}^{C} h^{\rho \sigma} m_{\sigma}^{D} \eta_{D B} \text {. }
\end{aligned}
$$

Let us further define $\hat{\tau}_{A}^{\mu}$ as

$\hat{\tau}_{A}^{\mu}=\tau_{A}^{\mu}-h^{\mu \rho} m_{\rho}^{B} \eta_{B A}$.

Then it is easy to see that

$$
\begin{aligned}
& \hat{\tau}_{A}^{\mu}\left(p_{\mu}-T \frac{\Gamma^{-}-\Gamma^{+}}{\Gamma^{+}+\Gamma^{-}} H_{\mu \nu} x^{\prime \nu}+T B_{\mu \nu} x^{\prime \nu}-\frac{T}{2} \lambda^{+} \tau_{\mu}-\frac{T}{2} \lambda^{-} \bar{\tau}_{\mu}\right) \\
& \quad=-\frac{2 T}{\Gamma^{+}+\Gamma^{-}} \Phi_{A B} \tau_{\nu}{ }^{B} \dot{x}^{\nu} .
\end{aligned}
$$

To proceed further we will presume that $\Phi_{A B}$ is non-singular matrix so that we can introduce its inverse in the form

$\left(\Phi^{-1}\right)^{A B}=\frac{1}{\operatorname{det} \Phi_{A B}}\left(\begin{array}{cc}\Phi_{11} & -\Phi_{01} \\ -\Phi_{01} & \Phi_{00}\end{array}\right)$

Now if we combine (28) with (31) we find that the inverse metric $H^{\mu \nu}$ to $H_{\mu \nu}$ has the form

$H^{\mu \nu} \equiv h^{\mu \nu}-\hat{\tau}_{A}^{\mu}\left(\Phi^{-1}\right)^{A B} \hat{\tau}_{B}^{\nu}, \quad H^{\mu \nu} H_{\nu \rho}=\delta_{\rho}^{\mu}$.

then it is easy to determine canonical Hamiltonian from (27) and we obtain

$$
\begin{aligned}
H= & \int d \sigma \mathcal{H}, \\
\mathcal{H}= & \frac{\left(\Gamma^{+}+\Gamma^{-}\right)}{4 T} \pi_{\mu} H^{\mu \nu} \pi_{v}+\frac{T}{4}\left(\Gamma^{+}+\Gamma^{-}\right) x^{\prime \mu} H_{\mu \nu} x^{\prime \nu} \\
& -\frac{1}{2}\left(\Gamma^{-}-\Gamma^{+}\right) x^{\prime \mu} \pi_{\mu} \\
& -\frac{1}{4}\left(\Gamma^{+}+\Gamma^{-}\right) \pi_{\mu} H^{\mu v}\left(\lambda^{+} \tau_{v}+\lambda^{-} \bar{\tau}_{v}\right) \\
& +\frac{T}{4}\left(\Gamma^{-}+\Gamma^{+}\right) x^{\prime \mu}\left(\lambda^{+} \tau_{\mu}-\lambda^{-} \bar{\tau}_{\mu}\right) \\
& +\frac{T}{16}\left(\Gamma^{+}+\Gamma^{-}\right)\left(\lambda^{+} \tau_{\mu}+\lambda^{-} \bar{\tau}_{\mu}\right) H^{\mu \nu}\left(\lambda^{-} \tau_{v}+\bar{\lambda}^{-} \tau_{v}\right),
\end{aligned}
$$

where

$\pi_{\mu}=p_{\mu}+T B_{\mu \rho} x^{\prime \rho}$.

Finally we introduce two variables $N$ and $N^{\sigma}$ defined as

$N=\frac{1}{4}\left(\Gamma^{+}+\Gamma^{-}\right), \quad N^{\sigma}=\frac{1}{2}\left(\Gamma^{+}-\Gamma^{-}\right)$

so that we find final form of the Hamiltonian density

$\mathcal{H}=N \mathcal{H}_{\tau}+N^{\sigma} \mathcal{H}_{\sigma}$

where

$$
\begin{aligned}
\mathcal{H}_{\tau}= & \frac{1}{T} \pi_{\mu} H^{\mu \nu} \pi_{v}+T x^{\prime \mu} H_{\mu \nu} x^{\prime \nu}-\pi_{\mu} H^{\mu \nu}\left(\lambda^{+} \tau_{v}+\lambda^{-} \bar{\tau}_{\nu}\right) \\
& +T x^{\prime \mu}\left(\lambda^{+} \tau_{\mu}-\lambda^{-} \bar{\tau}_{\mu}\right)
\end{aligned}
$$




$$
\begin{aligned}
& +\frac{T}{4}\left(\lambda^{+} \tau_{\mu}+\lambda^{-} \bar{\tau}_{\mu}\right) H^{\mu \nu}\left(\lambda^{-} \tau_{\nu}+\bar{\lambda}^{-} \tau_{\nu}\right), \\
\mathcal{H}_{\sigma}= & x^{\prime \mu} p_{\mu} .
\end{aligned}
$$

Let us now proceed to the analysis of the requirement of the preservation of all primary constraints. In case of the constraints $p_{N} \approx 0, p_{\sigma} \approx 0$ which are momenta conjugate to $N$ and $N^{\sigma}$ we obtain

$$
\begin{aligned}
& \dot{p}_{N}=\left\{p_{N}, H\right\}=-\mathcal{H}_{\tau} \approx 0, \\
& \dot{p}_{\sigma}=\left\{p_{\sigma}, H\right\}=-\mathcal{H}_{\sigma} \approx 0,
\end{aligned}
$$

while requirement of the preservation of the constraints $p_{+}^{\lambda} \approx$ $0, p_{-}^{\lambda} \approx 0$ implies

$$
\begin{aligned}
\dot{p}_{+}^{\lambda}= & \left\{p_{+}^{\lambda}, H\right\}=\pi_{\mu} H^{\mu v} \tau_{v}-T x^{\prime \mu} \tau_{\mu} \\
& -\frac{T}{2} \tau_{\mu} H^{\mu \nu}\left(\lambda^{-} \tau_{v}+\lambda^{-} \tau_{v}\right) \equiv \mathcal{G}_{+}^{\lambda} \approx 0, \\
\dot{p}_{-}^{\lambda}= & \left\{p_{-}^{\lambda}, H\right\}=\pi_{\mu} H^{\mu \nu} \bar{\tau}_{v}+T x^{\prime \mu} \bar{\tau}_{\mu} \\
& -\frac{T}{2} \bar{\tau}_{\mu} H^{\mu \nu}\left(\lambda^{-} \tau_{v}+\bar{\lambda}^{-} \bar{\tau}_{v}\right) \equiv \mathcal{G}_{-}^{\lambda} \approx 0 .
\end{aligned}
$$

Let us now analyze constraints $\mathcal{H}_{\sigma} \approx 0, \mathcal{H}_{\tau} \approx 0$ in more details. Since we can anticipate that $\mathcal{H}_{\sigma} \approx 0$ is generator of spatial diffeomorphism it is natural to extend it in the following way

$\mathcal{H}_{\sigma} \rightarrow p_{\mu} x^{\prime \mu}+\lambda^{\prime+} p_{+}^{\lambda}+\lambda^{\prime-} p_{-}^{\lambda}$

and introduce its smeared form

$\mathbf{T}_{\sigma}\left(N^{\sigma}\right)=\int d \sigma N^{\sigma} \mathcal{H}_{\sigma}$

together with smeared form of the Hamiltonian constraint $\mathbf{T}_{\tau}(N)=\int d \sigma N \mathcal{H}_{\tau}$. Note that $\mathbf{T}_{\sigma}\left(N^{\sigma}\right)$ has non-zero Poisson bracket with canonical variables

$$
\begin{aligned}
& \left\{\mathbf{T}_{\sigma}\left(N^{\sigma}\right), x^{\mu}\right\}=-N^{\sigma} x^{\prime \mu}, \\
& \left\{\mathbf{T}_{\sigma}\left(N^{\sigma}\right), p_{\mu}\right\}=-\left(N^{\sigma} p_{\mu}\right)^{\prime}, \\
& \left\{\mathbf{T}_{\sigma}\left(N^{\sigma}\right), \lambda^{ \pm}\right\}=-N^{\sigma} \lambda^{\prime \pm}, \\
& \left\{\mathbf{T}_{\sigma}\left(N^{\sigma}\right), p_{ \pm}^{\lambda}\right\}=-\left(N^{\sigma} p_{ \pm}^{\lambda}\right)^{\prime} .
\end{aligned}
$$

Then it is easy to see that

$$
\left\{\mathbf{T}_{\sigma}\left(N^{\sigma}\right), \mathbf{T}_{\sigma}\left(M^{\sigma}\right)\right\}=\mathbf{T}_{\sigma}\left(N^{\sigma} M^{\prime \sigma}-M^{\sigma} N^{\prime \sigma}\right) .
$$

In the same way we obtain

$$
\left\{\mathbf{T}_{\sigma}\left(N^{\sigma}\right), \mathcal{H}_{\tau}\right\}=-2 N^{\prime \sigma} \mathcal{H}_{\tau}-N^{\sigma} \mathcal{H}_{\tau}^{\prime}
$$

or equivalently

$$
\left\{\mathbf{T}_{\sigma}\left(N^{\sigma}\right), \mathbf{T}_{\tau}(M)\right\}=\mathbf{T}_{\tau}\left(N^{\sigma} M^{\prime}-M N^{\prime \sigma}\right) .
$$

Finally we calculate Poisson bracket

$$
\begin{aligned}
& \left\{\mathbf{T}_{\tau}(N), \mathbf{T}_{\tau}(M)\right\} \\
& =\int d \sigma\left(N M^{\prime}-M N^{\prime}\right)\left(p_{\mu} x^{\prime \mu}-2 x^{\prime \mu}\left(\lambda^{+} \tau_{\mu}+\lambda^{-} \bar{\tau}_{\mu}\right)\right. \\
& \left.\quad+2 \pi_{\mu} H^{\mu \nu}\left(\lambda^{+} \tau_{\nu}-\bar{\lambda}^{-} \bar{\tau}_{\nu}\right)\right) \\
& \quad-T\left(\lambda^{+} \tau_{\mu}-\bar{\lambda}^{-} \bar{\tau}_{\mu}\right) H^{\mu \nu}\left(\lambda^{+} \tau_{\nu}+\lambda^{-} \bar{\tau}_{\nu}\right) \\
& =\int d \sigma\left(N M^{\prime}-M N^{\prime}\right)\left(\mathcal{H}_{\sigma}+2\left(\lambda^{+} \mathcal{G}_{+}^{\lambda}-\lambda^{-} \mathcal{G}_{-}^{\lambda}\right)\right)
\end{aligned}
$$

that vanishes on the constraint surface $\mathcal{H}_{\sigma} \approx 0, \mathcal{G}_{+}^{\lambda} \approx$ $0, \mathcal{G}_{-}^{\lambda} \approx 0$. Collecting all these results together we find that $\mathcal{H}_{\tau} \approx 0, \mathcal{H}_{\sigma} \approx 0$ are the first class constraints which is an expected result since the action (8) defines relativistic theory on two-dimensional world-sheet $\Sigma$.

\section{Second class constraints and their solution}

Now we analyze the constraints $\mathcal{G}_{+}^{\lambda} \approx 0, \mathcal{G}_{-}^{\lambda} \approx 0$ in more details. First of all we show that $\mathcal{G}_{+}^{\lambda} \approx 0, \mathcal{G}_{-}^{\lambda} \approx 0$ are second class constraints together with $p_{+}^{\lambda} \approx 0$ and $p_{-}^{\lambda} \approx 0$ since

$\left\{p_{+}^{\lambda}(\sigma), \mathcal{G}_{+}^{\lambda}\left(\sigma^{\prime}\right)\right\}=\frac{T}{2} \tau_{\mu} H^{\mu \nu} \tau_{\nu} \delta\left(\sigma-\sigma^{\prime}\right)$,

$\left\{p_{+}^{\lambda}(\sigma), \mathcal{G}_{-}^{\lambda}\left(\sigma^{\prime}\right)\right\}=\frac{T}{2} \bar{\tau}_{\mu} H^{\mu \nu} \tau_{\nu} \delta\left(\sigma-\sigma^{\prime}\right)$,

$\left\{p_{-}^{\lambda}(\sigma), \mathcal{G}_{+}^{\lambda}\left(\sigma^{\prime}\right)\right\}=\frac{T}{2} \tau_{\mu} H^{\mu \nu} \bar{\tau}_{\nu} \delta\left(\sigma-\sigma^{\prime}\right)$,

$\left\{p_{-}^{\lambda}(\sigma), \mathcal{G}_{-}^{\lambda}\left(\sigma^{\prime}\right)\right\}=\frac{T}{2} \bar{\tau}_{\mu} H^{\mu \nu} \bar{\tau}_{\nu} \delta\left(\sigma-\sigma^{\prime}\right)$.

Clearly there is also non-zero Poisson bracket between $\mathcal{G}_{+}^{\lambda} \approx$ 0 and $\mathcal{G}_{-}^{\lambda} \approx 0$. Let us now introduce common notation for the second class constraint as $\Psi_{A} \equiv\left(p_{+}^{\lambda}, p_{-}^{\lambda}, \mathcal{G}_{+}^{\lambda}, \mathcal{G}_{-}^{\lambda}\right)$. Then the matrix of Poisson brackets between these constraints has schematic form

$\triangle_{A B}=\left(\begin{array}{cc}0 & X \\ Y & W\end{array}\right)$

where $X, Y, W$ are $2 \times 2$ matrices that have generally inverse matrices. ${ }^{4}$ Then the inverse matrix has the form

$\triangle^{A B}=\left(\begin{array}{cc}-Y^{-1} W X^{-1} & Y^{-1} \\ X^{-1} & 0\end{array}\right), \quad \triangle_{A B} \triangle^{B C}=\delta_{A}^{C}$.

If we now calculate Dirac bracket between $x^{\mu}$ and $p_{v}$ we obtain

${ }^{4}$ Of course, each entry of these matrices is infinite dimensional since it depends generally on $\sigma$ and $\sigma^{\prime}$. However for our purposes this schematic form is sufficient. 


$$
\begin{aligned}
& \left\{x^{\mu}, p_{\nu}\right\}_{D}=\left\{x^{\mu}, p_{\nu}\right\}-\left\{x^{\mu}, \Psi_{A}\right\} \triangle^{A B}\left\{\Psi_{B}, p_{\nu}\right\} \\
& =\left\{x^{\mu}, p_{\nu}\right\}-(0,0, *, *)\left(\begin{array}{cc}
-Y^{-1} W X^{-1} & Y^{-1} \\
X^{-1} & 0
\end{array}\right) \\
& \quad \times(0,0, *, *)^{T}=\left\{x^{\mu}, p_{\nu}\right\},
\end{aligned}
$$

where $*$ means non-zero entry whose explicit form is not important. From this result we see that Dirac brackets between $x^{\mu}$ and $p_{v}$ coincide with corresponding Poisson brackets. Now we are ready to solve the second class constraints $\mathcal{G}_{+}^{\lambda} \approx 0$ and $\mathcal{G}_{-}^{\lambda} \approx 0$. First of all we introduce part of the Hamiltonian constraint $\mathcal{H}_{\tau} \approx 0$ that depends on $\lambda^{+}$and $\lambda^{-}$as

$\mathcal{H}_{\tau}^{\lambda}=A \lambda^{+}+B \lambda^{-}+\frac{T}{4}\left(\left(\lambda^{+}\right)^{2} X+2 \lambda^{+} \lambda^{-} Y+\left(\lambda^{-}\right)^{2} W\right)$,

where

$A=-\pi_{\mu} H^{\mu v} \tau_{v}+T x^{\prime \mu} \tau_{\mu}, \quad B=-\pi_{\mu} H^{\mu \nu} \bar{\tau}_{\nu}-T x^{\prime \nu} \bar{\tau}_{\nu}$,

$X=\tau_{\mu} H^{\mu \nu} \tau_{\nu}, \quad Y=\bar{\tau}_{\mu} H^{\mu \nu} \tau_{\nu}, \quad W=\bar{\tau}_{\mu} H^{\mu \nu} \bar{\tau}_{\nu}$.

Using this notation we can write the solution of the second class constraints $\mathcal{G}_{+}^{\lambda}=0, \quad \mathcal{G}_{-}^{\lambda}=0$ in the form

$\lambda^{-}=-\frac{2}{T} \frac{A Y-B X}{Y^{2}-X W}, \quad \lambda^{+}=-\frac{2}{T} \frac{B Y-A W}{Y^{2}-X W}$.

Then inserting this result into $\mathcal{H}_{\tau}^{\lambda}$ we obtain

$\mathcal{H}_{\tau}^{\lambda}($ onshell $)=\frac{1}{T\left(Y^{2}-X W\right)}\left(A^{2} W+B^{2} X-2 A B Y\right)$,

where explicit calculations give

$X=-\left(\Phi^{-1}\right)^{00}-2\left(\Phi^{-1}\right)^{01}-\left(\Phi^{-1}\right)^{10}$,

$Y=\left(\Phi^{-1}\right)^{A B} \eta_{B A}$,

$W=-\left(\Phi^{-1}\right)^{00}+2\left(\Phi^{-1}\right)^{01}-\left(\Phi^{-1}\right)^{11}$

so that

$Y^{2}-X W=-\frac{4}{\operatorname{det} \Phi_{A B}}$.

Then after some complicated calculations and with the help of the explicit form of $A$ and $B$ given in (53) we get

$$
\begin{aligned}
& \mathcal{H}_{\tau}^{\lambda} \text { (onshell) } \\
& =\frac{1}{T} \pi_{\mu} \hat{\tau}_{A}^{\mu}\left(\Phi^{-1}\right)^{A B} \hat{\tau}_{B}^{\nu} \pi_{\nu}-2 \pi \mu \hat{\tau}_{A}^{\mu} \epsilon^{A B} \eta_{B C} \tau_{\sigma}{ }^{C} \\
& \quad+T \tau_{\sigma}{ }^{A} \Phi_{A B} \tau_{\sigma}{ }^{B}-T \tau_{\sigma}{ }^{A} \tau_{\sigma}{ }^{B} \eta_{A B} \Phi_{C D} \eta^{C D},
\end{aligned}
$$

where $\tau_{\sigma}{ }^{A} \equiv x^{\prime \mu} \tau_{\mu}{ }^{A}$. Inserting (58) into (38) we obtain Hamiltonian constraint that depends on the canonical variables $x^{\mu}$ and $p_{\mu}$ only

$$
\begin{aligned}
\mathcal{H}_{\tau}^{\text {sol }}= & \frac{1}{T} \pi_{\mu} h^{\mu v} p_{\nu}+T x^{\prime \mu} H_{\mu \nu} x^{\prime \nu} \\
& -2 \pi \mu \hat{\tau}^{\mu}{ }^{A} \epsilon^{A B} \eta_{B C} \tau_{\sigma}{ }^{C} \\
& +T \tau_{\sigma}{ }^{A} \Phi_{A B} \tau_{\sigma}{ }^{B}-T \tau_{\sigma}{ }^{A} \tau_{\sigma}{ }^{B} \eta_{A B} \Phi_{C D} \eta^{C D},
\end{aligned}
$$

where $\tau_{\sigma}{ }^{A} \equiv x^{\prime \mu} \tau_{\mu}{ }^{A}$. The form of the Hamiltonian constraint (59) coincides with the Hamiltonian constraint found in [26] where non-relativistic string in stringy Newton-Cartan background was defined with the help of the limiting procedure that defines Newton-Cartan geometry from the relativistic one. We mean that this is very nice consistency check of both approaches.

Finally we would like to check the analysis further and try to determine corresponding Lagrangian density. Using canonical equation of motion we get

$$
\dot{x}^{\mu}=\left\{x^{\mu}, H\right\}=\frac{2 N}{T} h^{\mu \nu} \pi_{v}-2 N \hat{\tau}_{A}^{\mu} \epsilon^{A B} \eta_{B C} \tau_{\sigma}{ }^{C}
$$$$
+N^{\sigma} \partial_{\sigma} x^{\mu} \text {, }
$$

where $H=\int d^{\sigma}\left(N \mathcal{H}_{\tau}^{s o l}+N^{\sigma} \mathcal{H}_{\sigma}\right)$. Then we find

$$
\begin{aligned}
\mathcal{L}= & p_{\mu} \dot{x}^{\mu}-N \mathcal{H}_{\tau}^{\text {sol }}-N^{\sigma} \mathcal{H}_{\sigma} \\
= & \frac{N}{T} \pi_{\mu} h^{\mu \nu} \pi_{\nu}-T \tau_{\sigma}{ }^{A} \Phi_{A B} \tau_{\sigma}{ }^{B}+T \tau_{\sigma}{ }^{A} \tau_{\sigma}{ }^{B} \Phi_{A B} \\
& -T x^{\prime \mu} H_{\mu \nu} x^{\prime \nu}-T \partial_{\tau} x^{\mu} B_{\mu \nu} \partial_{\sigma} x^{\nu} .
\end{aligned}
$$

To proceed further we will now follow [26] and introduce $\hat{E}_{\mu}^{A^{\prime}}$ defined as

$\hat{E}_{\mu}^{A^{\prime}}=E_{\mu}^{A^{\prime}}+m_{\nu}{ }^{A} E^{v}{ }_{C^{\prime}} \delta^{C^{\prime} A^{\prime}} \tau_{\mu}{ }^{B} \eta_{B A}$

that obeys an important relation

$\hat{E}_{\mu}^{A^{\prime}} \hat{\tau}_{B}^{\mu}=0$

Then it is easy to express Lagrangian density (61) as function of $x^{\mu}$ and $\partial_{\alpha} x^{\mu}$ and we obtain

$$
\begin{aligned}
\mathcal{L}= & \frac{T}{4 N}\left(\dot{x}^{\mu}-N^{\sigma} x^{\prime \mu}\right) \hat{E}_{\mu}^{A^{\prime}} \delta_{A^{\prime} B^{\prime}} \hat{E}_{\nu}^{B^{\prime}}\left(\dot{x}^{\nu}-N^{\sigma} x^{\prime \nu}\right) \\
& -T N \tau_{\sigma}^{A} \Phi_{A B} \tau_{\sigma}{ }^{B}+T N \tau_{\sigma}{ }^{A} \tau_{\sigma}{ }^{B} \eta_{A B} \Phi_{C D} \eta^{C D} \\
& -T N x^{\prime \mu} H_{\mu \nu} x^{\prime \nu}-T \partial_{\tau} x^{\mu} B_{\mu \nu} \partial_{\sigma} x^{\nu} .
\end{aligned}
$$

As the next step we determine Lagrange multipliers $N$ and $N^{\sigma}$. It turns out that these multipliers are determined by equations of motion for $x^{\mu}$. In fact, if we multiply this equation by $\tau_{\mu \nu}$ we obtain

$$
\tau_{\mu \nu}\left(\dot{x}^{\nu}-N^{\sigma} x^{\prime \nu}\right)=-2 N \tau_{\mu}^{E} \epsilon_{E D} \tau_{\sigma}^{D} .
$$

If we further multiply this result with $x^{\prime \mu}$ and use an antisymmetry of $\epsilon_{A B}$ we obtain

$$
N^{\sigma}=\frac{\tau_{\tau \sigma}}{\tau_{\sigma \sigma}} .
$$


If we manipulate with (65) further we get

$\left(\dot{x}^{\mu}-N^{\sigma} x^{\prime \mu}\right) \tau_{\mu \nu}\left(\dot{x}^{\nu}-N^{\sigma} x^{\prime \nu}\right)=-4 N^{2} \tau_{\sigma \sigma}$

that can be written as

$\tau_{\tau \tau}-2 N^{\sigma} \tau_{\sigma \tau}+\left(N^{\sigma}\right)^{2} \tau_{\sigma \sigma}=-4 N^{2} \tau_{\sigma \sigma}$

This equation can be solved for $N$ when we take into account the result (66) and we obtain

$N=\frac{1}{2} \frac{\sqrt{-\operatorname{det} \tau_{\alpha \beta}}}{\tau_{\sigma \sigma}}$.

Inserting (66) and (69) into (64) we obtain Lagrangian density in the form

$$
\begin{aligned}
\mathcal{L}= & -\frac{T}{2} \sqrt{-\operatorname{det} \tau_{\alpha \beta}} \tau^{\alpha \beta} H_{\alpha \beta}-\frac{T}{2} \sqrt{-\operatorname{det} \tau_{\alpha \beta}} \tau^{\alpha \beta} \tau_{\alpha}{ }^{A} \Phi_{A B} \tau_{\beta}{ }^{B} \\
& +\frac{T}{2} \sqrt{-\operatorname{det} \tau_{\alpha \beta}} \Phi_{A B} \eta^{A B}-T \dot{x}^{\mu} B_{\mu \nu} x^{\prime \nu}
\end{aligned}
$$

where $H_{\alpha \beta}=H_{\mu \nu} \partial_{\alpha} x^{\mu} \partial_{\beta} x^{\nu}, \tau_{\alpha}{ }^{A}=\partial_{\alpha} x^{\mu} \tau_{\mu}{ }^{A}$ and where we used the fact that

$\hat{E}_{\mu}^{A^{\prime}} \delta_{A^{\prime} B^{\prime}} \hat{E}_{v}^{B^{\prime}}=H_{\mu \nu}+\tau_{\mu}^{A} \Phi_{A B} \tau_{v}{ }^{B}$.

We see that this Lagrangian density almost coincides with the Lagrangian density found [18] up to terms that contain matrix valued Newton potential $\Phi_{A B}$. Now we are going to argue that these terms cancel each other. In fact, note that $\tau_{\alpha \beta}$ is defined as

$\tau_{\alpha \beta}=\tau_{\alpha}{ }^{A} \tau_{\beta}{ }^{B} \eta_{A B}$,

where $\tau_{\alpha}{ }^{A}$ is $2 \times 2$ matrix. Now since $\tau_{\alpha \beta}$ is non-singular so that $\tau_{\alpha}{ }^{A}$ is non-singular as well and hence we can introduce an inverse matrix $\tau_{A}^{\beta}$ that obeys the relation

$\tau_{A}^{\alpha} \tau_{\alpha}^{B}=\delta_{A}^{B}$

Then we can define $\tau^{\alpha \beta}$ as

$\tau^{\alpha \beta}=\tau_{A}^{\alpha} \tau_{B}^{\beta} \eta_{A B}$

that obeys

$\tau^{\alpha \beta} \tau_{\beta}^{A}=\tau_{C}^{\alpha} \eta^{C A}$

and hence

$\tau^{\alpha \beta} \tau_{\beta}^{B} \tau_{\alpha}^{A}=\tau_{\beta}^{B} \tau_{C}^{\beta} \eta^{C A}=\eta^{B A}$.

With the help of these results it is easy to see that contributions to the Lagrangian density (70) that depend on $\Phi_{A B}$ cancel each other and hence the Lagrangian density has the final form

$\mathcal{L}=-\frac{T}{2} \sqrt{-\operatorname{det} \tau_{\alpha \beta}} \tau^{\alpha \beta} H_{\alpha \beta}-T \dot{x}^{\mu} B_{\mu \nu} x^{\prime \nu}$

which is Lagrangian density proposed in [18]. This result again confirms validity of our approach.

Acknowledgements This work was supported by the Grant Agency of the Czech Republic under the Grant P201/12/G028.

Data Availability Statement This manuscript has no associated data or the data will not be deposited. [Authors' comment: This is a theoretical study and no experimental data has been listed.]

Open Access This article is distributed under the terms of the Creative Commons Attribution 4.0 International License (http://creativecomm ons.org/licenses/by/4.0/), which permits unrestricted use, distribution, and reproduction in any medium, provided you give appropriate credit to the original author(s) and the source, provide a link to the Creative Commons license, and indicate if changes were made.

Funded by $\mathrm{SCOAP}^{3}$.

\section{References}

1. J. Gomis, H. Ooguri, Nonrelativistic closed string theory. J. Math. Phys. 42, 3127 (2001). https://doi.org/10.1063/1.1372697. arXiv:hep-th/0009181

2. U.H. Danielsson, A. Guijosa, M. Kruczenski, IIA/B, wound and wrapped. J. High Energy Phys. 0010, 020 (2000). https://doi.org/ 10.1088/1126-6708/2000/10/020. arXiv:hep-th/0009182

3. J. Gomis, P.K. Townsend, The Galilean superstring. J. High Energy Phys. 1702, 105 (2017). https://doi.org/10.1007/ JHEP02(2017)105. arXiv:1612.02759 [hep-th]

4. C. Batlle, J. Gomis, D. Not, Extended Galilean symmetries of nonrelativistic strings. J. High Energy Phys. 1702, 049 (2017). https:// doi.org/10.1007/JHEP02(2017)049. arXiv:1611.00026 [hep-th]

5. M. Sakaguchi, K. Yoshida, Holography of non-relativistic string on AdS(5) x S**5. J. High Energy Phys. 0802, 092 (2008). https://doi. org/10.1088/1126-6708/2008/02/092. arXiv:0712.4112 [hep-th]

6. R. Casalbuoni, J. Gomis, G. Longhi, Exotic non-relativistic string. Phys. Rev. D 76, 126004 (2007). https://doi.org/10.1103/ PhysRevD.76.126004. arXiv:0709.4385 [hep-th]

7. B.S. Kim, World sheet commuting beta gamma CFT and nonrelativistic string theories. Phys. Rev. D 76, 106007 (2007). https:// doi.org/10.1103/PhysRevD.76.106007. arXiv:0708.4261 [hep-th]

8. M. Sakaguchi, K. Yoshida, Non-relativistic string and D-branes on $\operatorname{AdS}(5) \times S^{* *} 5$ from semiclassical approximation. J. High Energy Phys. 0705, 051 (2007). https://doi.org/10.1088/1126-6708/2007/ 05/051. arXiv:hep-th/0703061 [HEP-TH]

9. J. Kluson, Non-relativistic non-BPS Dp-brane. Nucl. Phys. B 765, 185 (2007). https://doi.org/10.1016/j.nuclphysb.2006.12.010. arXiv:hep-th/0610073

10. M. Sakaguchi, K. Yoshida, Non-relativistic AdS branes and Newton-Hooke superalgebra. J. High Energy Phys. 0610, 078 (2006). https://doi.org/10.1088/1126-6708/2006/10/ 078. arXiv:hep-th/0605124

11. J. Brugues, J. Gomis, K. Kamimura, Newton-Hooke algebras, nonrelativistic branes and generalized pp-wave metrics. Phys. Rev. D 73, 085011 (2006). https://doi.org/10.1103/PhysRevD.73.085011. arXiv:hep-th/0603023 
12. J. Gomis, F. Passerini, T. Ramirez, A. Van Proeyen, Non relativistic Dp branes. J. High Energy Phys. 0510, 007 (2005). https://doi.org/ 10.1088/1126-6708/2005/10/007. arXiv:hep-th/0507135

13. J. Gomis, J. Gomis, K. Kamimura, Non-relativistic superstrings: a new soluble sector of $\operatorname{AdS}(5) \times S^{* * 5}$. J. High Energy Phys. 0512, 024 (2005). https://doi.org/10.1088/1126-6708/2005/12/ 024. arXiv:hep-th/0507036

14. J. Gomis, F. Passerini, Rotating solutions of non-relativistic string theory. Phys. Lett. B 617, 182 (2005). https://doi.org/10.1016/j. physletb.2005.04.061. arXiv:hep-th/0411195

15. J. Gomis, K. Kamimura, P.K. Townsend, Non-relativistic superbranes. J. High Energy Phys. 0411, 051 (2004). https://doi.org/10. 1088/1126-6708/2004/11/051. arXiv:hep-th/0409219

16. J. Brugues, T. Curtright, J. Gomis, L. Mezincescu, Non-relativistic strings and branes as non-linear realizations of Galilei groups. Phys. Lett. B 594, 227 (2004). https://doi.org/10.1016/j.physletb.2004. 05.024. arXiv:hep-th/0404175

17. E. Bergshoeff, J. Gomis, Z. Yan, Nonrelativistic string theory and T-duality. arXiv:1806.06071 [hep-th]

18. R. Andringa, E. Bergshoeff, J. Gomis, M. de Roo, 'Stringy' Newton-Cartan gravity. Class. Quant. Gravit. 29, 235020 (2012). $\quad$ https://doi.org/10.1088/0264-9381/29/23/235020. arXiv: 1206.5176 [hep-th]

19. J. Kluso, Remark about non-relativistic string in Newton*Cartan background and null reduction. J. High Energy Phys. 1805, 041 (2018). https://doi.org/10.1007/JHEP05(2018)041. arXiv:1803.07336 [hep-th]

20. J. Kluson, Note about Hamiltonian formalism for NewtonCartan string and p-brane. Eur. Phys. J. C 78(6), 511 (2018). https://doi. org/10.1140/epjc/s10052-018-5993-8. arXiv:1712.07430 [hep-th]

21. A. Barducci, R. Casalbuoni, J. Gomis, Non-relativistic spinning particle in a Newton-Cartan background. J. High Energy Phys. 1801, 002 (2018). https://doi.org/10.1007/JHEP01(2018)002. arXiv: 1710.10970 [hep-th]
22. J. Kluson, Canonical analysis of non-relativistic particle and superparticle. Eur. Phys. J. C 78(2), 117 (2018). https://doi.org/10.1140/ epjc/s10052-018-5609-3. arXiv:1709.09405 [hep-th]

23. J. Kluson, Remark about non-relativistic p-brane. Eur Phys. J. C 78(1), 27 (2018). https://doi.org/10.1140/epjc/ s10052-017-5500-7. arXiv:1707.04034 [hep-th]

24. T. Harmark, J. Hartong, N.A. Obers, Nonrelativistic strings and limits of the AdS/CFT correspondence. Phys. Rev. D 96(8), 086019 (2017). https://doi.org/10.1103/PhysRevD.96. 086019. arXiv:1705.03535 [hep-th]

25. J. Kluson, Hamiltonian analysis of non-relativistic non-BPS Dpbrane. J. High Energy Phys. 1707, 007 (2017). https://doi.org/10. 1007/JHEP07(2017)007. arXiv:1704.08003 [hep-th]

26. J. Kluson, Hamiltonian for string in Newton-Cartan background. arXiv:1801.10376 [hep-th]

27. E. Bergshoeff, J. Rosseel, T. Zojer, NewtonCartan (super)gravity as a non-relativistic limit. Class. Quant. Gravit. 32(20), 205003 (2015). $\quad$ https://doi.org/10.1088/0264-9381/32/20/205003. arXiv: 1505.02095 [hep-th]

28. T. Fujiwara, H. Igarashi, T. Suzuki, Super-Virasoro anomaly, superWeyl Anomaly, and the super-Liouville action for 2D supergravity. Ann. Phys. 254, 233 (1997). https://doi.org/10.1006/aphy.1996. 5643. [arXiv:hep-th/9605138]

29. T. Fujiwara, Y. Igarashi, R. Kuriki, T. Tabei, BFV-BRST quantization of 2-D supergravity. Phys. Rev. D 53, 852 (1996). https://doi. org/10.1103/PhysRevD.53.852. arXiv:hep-th/9502127 